\title{
Effectiveness of 14 days concomitant therapy as a new regimen in treatment of Helicobacter pylori infection on a sample of Lebanese population
}

\author{
Khalil Ali, Hallal Mahmoud, Mahfouz Rayane, Moubarak Zeinab, Alomary Abdallah, Assaf Moushira and Matar Rasha* \\ Department of Medicine, Division of Gastroenterology, Al Zahraa Hospital University Medical Center, Jnah, Beirut, Lebanon
}

\begin{abstract}
Background: Finding new efficacious regimens for H Pylori eradication remains the goal of many studies especially after the increased resistance to standard therapy, which includes clarithromycin as the main antibiotic. In the current study, the aim is to evaluate the efficacy of concomitant or non-bismuth quadruple therapy in eradication of H Pylori.

Methods: Fifty patients from Al-Zahraa Hospital University Medical Center (ZHUMC) receiving concomitant therapy were included in the study from August 2015 to December 2016. Patients were presented with symptoms of gastritis, peptic ulcer diseases (epigastric pain, heartburn, halitosis, nausea) and found to have $H$. pylori infection through Rapid Urease Test (RUT) following endoscopy, were included in this study. Patients were assigned into the 14 days' concomitant non-bismuth quadruple therapy, which is consisted of Lansoprazole (30 mg), amoxicillin ( $1 \mathrm{~g})$, clarithromycin (500 mg) and metronidazole (500 mg). Our primary endpoint was H. Pylori eradication as established by a negative urease breath test at least 2 weeks after the end of treatment.
\end{abstract}

Results: All were evaluated at the end of the study. The $H$. pylori eradication rate was $94 \%$ (47/50). The treatment was well tolerated by the participants to the study with a $96 \%$ compliance.

Conclusion: A total 14-day concomitant therapy is a highly effective treatment for $H$. pylori infection as showed by the high tolerance, mild and non-serious side effects in our sample of patients.

\section{Introduction}

Between many stomach pathogens, Helicobacter Pylori (H.pylori) is defined as a microaerophilic stomach pathogen which infects the half of the world's population in a chronic manner [1]. It is responsible for many diseases ranging from mild ones such as asymptomatic gastritis to more severe ones like stomach and duodenal ulcers in $10 \%$ of patients and stomach cancers in $1 \%$. H. pylori plays a major role in the pathogenesis of chronic gastritis, gastro-duodenal ulcers, gastric mucosa-associated lymphoid tissue lymphoma, and gastric malignancies [2].

Despite the use of antibiotics in many regimens and the improved hygiene conditions which reduced the prevalence of H.Pylori ; the infection incidence remains high on a global scale [3]. The worldwide resistance to antibiotics reduces their effectiveness in the H.Pylori treatment [4].

Many therapies were used to eradicate H.Pylori: triple-therapy, sequential-therapy, non-bismuth concomitant therapy, bismuth quadruple therapy. The efficacy of the first triple treatment of $H$. pylori (PPI, clarithromycin, amoxicillin or metronidazole) has been declined to $70 \%$ eradication rate due to the increased prevalence of clarithromycin resistance [5,6]. For this reason, the Maastricht III guidelines recommends the use of first-line triple therapy in regions with clarithromycin resistance lower than $15 \%$ to $20 \%$ [6]. Different combinations of known antibiotics were used in the treatment of H.pylori under the name of sequential therapy.

Clarithromycin is a macrolide antibiotic that inhibits protein synthesis by binding to and slowing the actions of the bacterial ribosome. Clarithromycin resistance is due to three-point mutations at A2142C, A2142G, and A2143G in the 23s rRNA gene. In particular, the A2143G mutation has been related to a very low eradication rate. In contrast, the $\mathrm{A} 2143 \mathrm{G}$ mutation occurs in only $23 \%$ of resistant strains in Eastern countries. This suggests that clarithromycin point mutations may be geographically distinct between Eastern and Western countries (7). Among Eastern countries a high resistance to clarithromycin has been noted for example in South Korea, it ranges from $17.2 \%$ to $23.7 \%$ [6], in Japan, it is of $18.9 \%$ to $27.2 \%$ [6]. Even in the western countries , low resistance was been noted in northern Europe .

In Lebanon, data related to the prevalence of $H$. pylori resistance to clarithromycin are poor, since the last study concerning this subject was done in 2002 and showed a resistance rate of 4\% [6].

Despite the relationship between geographic area and prevalence of clarithromycin resistance, the overall frequency of clarithromycin resistance continues to rise from $10.2 \%$ to $21.3 \%$ worldwide with A2143G identified as the most frequently reported point mutation [7].

Metronidazole resistance may also influence the treatment outcome, although it is generally considered less clinically important

*Correspondence to: MMatar Rasha, Department of Medicine, Division of Gastroenterology, Al Zahraa Hospital University Medical Center, Jnah, Beirut, Lebanon, Tel: +961-1-851 040, E-mail: rasha.matar@yahoo.com

Received: November 09, 2020; Accepted: November 24, 2020; Published: November 27, 2020 
than clarithromycin resistance. Overall, due to higher consumption rates of these antibiotics the metronidazole resistance rates were $95.4 \%$ in Eastern Asian region, and $71.3 \%$ in the southeast costal region of China in Japan [7].

Amoxicillin is a beta-lactam antibiotic that was first used for $H$. pylori therapy. Unlike clarithromycin and metronidazole, amoxicillin resistance rates are low worldwide [7].

The choice of treatment regimen, the patient compliance to multidrug regimen with side effects and the sensitivity of H.pylori strains to antibiotics of choice are the main factors that can predict the treatment outcome [8].

In intention-to-treat analysis, an eradication rate of $88 \%$ was confirmed by the use of first-line concomitant therapy consisting of PPI, amoxicillin, and clarithromycin plus either metronidazole or tinidazole [9].

A meta-analysis comparing concomitant, sequential and triple therapy showed that the concomitant therapy can be an effective alternative to triple therapy and less complex than sequential therapy [10]. Consequently, the Maastricht guidelines recommended concomitant therapy as an alternative first-line therapy for $H$. pylori eradication in areas where clarithromycin resistance is more than $20 \%$ [11]. The quadruple therapy used over 10 days showed efficacy in areas with metronidazole resistance in North America with $91.7 \%$ eradication in metronidazole-sensitive strains and $80.4 \%$ eradication in metronidazole-resistant strains [12].

Using the quadruple therapy for 10 days showed $95 \%$ eradication of strains resistant to metronidazole and $99 \%$ eradication of strains sensitive to metronidazole (by per protocol analysis) which justify that the metronidazole resistance did not influence the efficacy of quadruple therapy [13]. Moreover, although this regimen has produced one of the highest cure rates, patients perceive it as complex due to the numerous pills taken per day. This poses the problem of therapeutic non-compliance.

\section{Methods and results}

This is a 10-month prospective randomized study concerning eradication of $\mathrm{H}$. pylori with concomitant non bismuth quadruple therapy based on data collected from patients admitted to AL-Zahraa Hospital University Medical Center (ZHUMC) from August 2015 to December 2016.

The population included in this study consists of subjects admitted to ZHUMC in this period of time with Upper Gastrointestinal symptoms (dyspepsia and epigastric pain) with or without ulcer disease, and confirmed positive for H.pylori infection. The patients were selected according to the following inclusion criteria:

- Both genders

- Aged between 17 and 75 years

- Naive to H. pylori treatment.

Patients excluded from our study are:

- Women who were pregnant or lactating

- Patients previously treated with $H$. pylori eradication therapy

- Patients who previously underwent gastric surgery

- Patients with malignant neoplasms
- Patients with other severe concomitant diseases (CAD; HTN; diabetes...)

- Gastric malignancy, including adenocarcinoma and lymphoma

- Previous allergic reaction

History was taken from all patients that were also subjected to abdominal physical examination and the majority of patients had epigastric tenderness. Diagnosis of H. Pylori infection is obtained using an endoscopic biopsy-based method: RUT. In fact, subjects underwent upper GI endoscopy to obtain gastric biopsies: one biopsy from the antrum and another from the body of the stomach, under sedation. The collected biopsy specimens were then sent for examination for $H$. pylori using the RUT. This test consists of an agar gel containing phenol red and urea. In the presence of $H$. pylori urease, urea is hydrolyzed to ammonia, leading to a rise in $\mathrm{pH}$ and color change from yellow to red of the $\mathrm{pH}$ indicator (phenol red). Due to the high specificity of this test (99-100\%), patients with positive results are considered infected with $\mathrm{H}$. Pylori.

After documentation of the infection, a clear explanation of the purpose and risks of the study was given to the patients. Those who agreed to participate were enrolled in our study and they received the quadruple therapy regimen.

The study drug consisted of the concomitant "non-bismuth' quadruple therapy which is consisted of: Lansoprazole $30 \mathrm{mg}$ bid, Amoxicillin $1 \mathrm{~g}$ bid, clarithromycin $500 \mathrm{mg}$ bid and metronidazole 500 mg bid.

Eligible patients received the 4 capsules, twice daily after meals for the whole 14-day period. Afterwards, they were started on a Single dose of PPI for one month.

Patients returned for follow-up visit at the end of the eradication therapy (on day 15 after starting the quadruple therapy) to assess their compliance and evaluate any possible side effects. Patients were informed that a UBT will be performed starting from day 41 after the follow-up visit.

The efficacy of the treatment and H pylori eradication were assessed 55 days after the end of the treatment. In fact, they were subjected to the UBT, which should be negative if the treatment was efficient. Before performing ${ }^{13} \mathrm{C}$ labelled urea breath test, patients were asked to avoid PPI treatment for 2 weeks and antibiotics for 1 month respectively. After an overnight fast, each patient was asked to blow into a tube to obtain the baseline breath sample, then to drink a test meal $\left({ }^{13} \mathrm{C}\right.$-labelled urea solution) and blow again into the tube 15 minutes after drinking the solution to obtain the second breath sample.

All of the breath samples were analysed by an isotope ratio mass spectrometer by a technician who was blinded to treatment.

The data analysis was conducted using the SPSS (Statistical Package for the Social Sciences) 21.1 version for windows. The eradication rate will be evaluated directly using the quotient of the patients with negative UBT over the total number of patients. Association between categorical variables such as age and sex, and symptoms with the eradication of H.Pylori were determined using 95\% confidence interval (95\% CI). The p-values less than 0.05 were considered statistically significant.

These results were collected by the descriptive statistics and the correlation analysis test, which consists of a statistical technique that can show whether and how strongly pairs of variables are related. The p-values were calculated using Pearson test correlation coefficient which is significant at 0.05 level. 


\section{Demographic analysis and urea breathe test results}

50 patients fulfilling the inclusion and exclusion criteria were enrolled in the study. These subjects were assigned to receive concomitant non-bismuth quadruple therapy and to complete the protocols in full compliance with the study rules. The mean age of the enrolled subjects was $44.12 \pm 15.709$ years; 20 males (40\%) and 30 females (60\%) with a range from 17 to 75 years (Table 1 ). After completing the $H$. pylori eradication regimen, all the 50 patients returned to undergo the control test (Urea Breath Test) to confirm $H$. pylori status 55 days after completing treatment (Table 2).

\section{Eradication rate of $\boldsymbol{H}$. Pylori}

Since the all the participants assigned to the treatment have completed the study rules and the treatment regimen (described in the previous section and in the Figure 1), the per protocol and the intention-to- treat eradication rates achieved in our sample are the same: $94 \%(47 / 50)$.

\section{Compliance, tolerability and side effects}

We achieved $96 \%$ compliance with the regimen (two patients were not compliant to regimen because they skipped some drug doses and has positive UBT).

Drugs in general were well tolerated in the majority of the patients and no severe side effects nor troubles in daily activities were reported.

\section{Association between eradication rate and gender}

The concomitant non-bismuth quadruple therapy results for $H$. pylori eradication were almost similar between women $93 \%(28 / 30)$ and men $95 \%$ (19/20), regardless of their age. This small difference was statistically insignificant with $\mathrm{p}$ value 0.813 ( $\mathrm{P}$ value $>0.05$ ).

This result shows that gender had no effect on the eradication rate in our study.

\section{Association between eradication rate and age}

To study the association between age and eradication rate, we calculated the mean ages of the patients with negative UBT $42.3 \pm 14.3$ years and those with positive UBT $72.6 \pm 2.31$ years.

$P$ value was $0.001(<0.05)$ implicating that the eradication rate is significantly affected by age. And in order to clarify which age range had affected more the failure of the treatment, the eradication rate

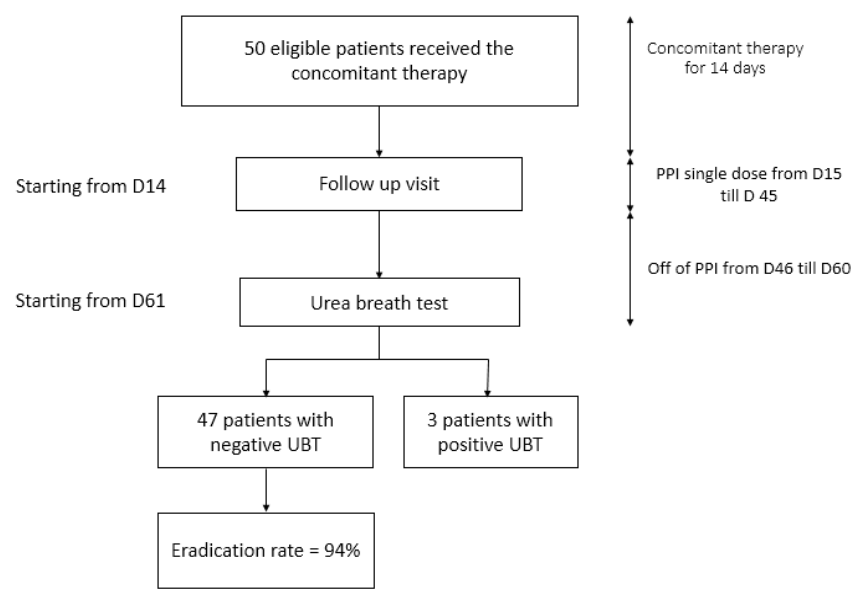

Figure 1. Flow diaphragm of study design with repartition of treatment over time
Table 1. Demographic data

\begin{tabular}{|c|c|}
\hline & Number of patients \\
\hline Age & 13 \\
$17-30$ & 20 \\
$31-50$ & 17 \\
$51-75$ & \\
\hline Gender & 20 \\
Male & 30 \\
\hline Female & \\
\hline
\end{tabular}

Table 2. UBT results and compliance

\begin{tabular}{|c|c|}
\hline & Number of patients \\
\hline UBT status & 3 \\
Positive & 47 \\
Negative & \\
\hline Compliance & 48 \\
Yes & 2 \\
No & \\
\hline
\end{tabular}

Table 3. Age and gender distribution of the study participants

\begin{tabular}{|l|c|c|c|c|c|c|}
\hline \multicolumn{3}{|c|}{} & \multicolumn{3}{|c|}{ Men } & \multicolumn{3}{c|}{ Women } \\
\hline Age & $17-30$ & $30-50$ & $50-75$ & $17-30$ & $30-50$ & $50-75$ \\
\hline Positive UBT & 4 & 9 & 6 & 9 & 11 & 8 \\
\hline Negative UBT & 0 & 0 & 1 & 0 & 0 & 2 \\
\hline Total & 4 & 9 & 7 & 9 & 11 & 10 \\
\hline
\end{tabular}

corresponding to each age range was calculated using table 3 and the results were as follows: $100 \%(13 / 13)$ for the young category (17 to 30 years old), $100 \%(20 / 20)$ for the middle-aged category (30 to 50 years old) and $82.3 \%(14 / 17)$ for the older patients (50 to 75 years old).

Consequently, in our study, only advanced ages were associated with a lower rate of $H$. pylori eradication. P value (0.033) was significant upon correlation between age group and positive UBT. This result shows that the age has a negative effect on eradication rate. In addition, in all age groups, there was no significant difference in $H$. pylori eradication rate between genders with a $\mathrm{P}$ value of 0.09 (Table 3).

\section{Discussion}

This study was intended to evaluate the ability of the concomitant non-bismuth quadruple therapy, to eradicate $H$. pylori as a first-line treatment; as well as the compliance associated with this regimen. The evaluation of the eradication rate of $H$. pylori using amoxicillin, metronidazole, clarithromycin combined with PPI was the primary objective of this study. Since all the participants in this study fulfilled the protocol in the terms of the eligibility, interventions and outcome assessment, the eradication rate by both ITT and PP analyses was $94 \%$, a percentage high enough to show the efficacy of this regimen against the $H$. pylori infection.

This study confirms the results obtained with this drug regimen in different clinical trials of different treatment durations. A study compared 480 patients treated with concomitant therapy and 370 patients treated with hybrid therapy showed an eradication rate of $86.7 \%$ with concomitant therapy and $84.9 \%$ for hybrid therapy [14].

Moreover, five qualifying RCTs including 576 subjects where 293 subjects received concomitant therapy for 3 to 5 days and 283 subjects received triple therapy for 5 to 10 days showed superiority of concomitant therapy over triple therapy with odds ratio 2.86 vs 3.52 respectively [15].

On the other hand, a study evaluating concomitant therapy for 3 to 7 days showed eradication rate of $92.9 \%$ and mentioned that the concomitant therapy appears to be an effective alternative to triple therapy and is less complex than sequential therapy [15]. 
In regions with higher rates of resistance to clarithromycin or metronidazole the quadruple therapy is considered an efficacious regimen for the treatment of H.pylori or for treatment of patients who are at a heightened risk of resistance to these antibiotics [16].

In addition, a randomized controlled trial showed a better eradication rate with concomitant therapy compared to sequential therapy with intention-to-treat (ITT), 79.4\% versus $70.7 \%$, and perprotocol (PP), $94.0 \%$ versus $84.4 \%$ [17].

Thereafter those two therapies given concomitantly or sequentially, showed better eradication rate in comparison to the standard triple therapy [17].

In addition to the ability of the concomitant therapy applied for 14 days in the cure of more than $90 \%$ of patients with $H$. Pylori infections in areas of high clarithromycin and metronidazole resistance, the appliance of all four drugs concomitantly for the entire therapy duration, indicating that this regimen is less complex than sequential therapy because it does not involve changing the number or type of drugs halfway through the therapy [18].

That resistance to metronidazole and clarithromycin which considerably influences sequential therapy did not affect the success rate of concomitant quadruple therapy [19]. This finding has been confirmed by a study which showed an eradication rate of $100 \%$ vs $75 \%$ for clarithromycin-resistant strains and 75\% vs 60\% for clarithromycinresistant/metronidazole resistant strains in a group of patients treated with concomitant and another one treated with sequential therapy respectively [18].

In addition, the concomitant regimen is superior to sequential therapy in term of safety and efficacy with an eradication rate of 23.5 $\%$ in an areas with high resistance to clarithromycin, 33\% in areas with resistant strains of H.pylori to metronidazole and $8.8 \%$ in areas with strains resistant to both drugs [19].

Compliance is a primary determinant of treatment success. On the one hand, the compliance rate in general depends on the quality of the provider-patient communication. On the other hand, it is tightly linked to the duration and complexity of treatment [20].

In our study, the mean compliance rate with the quadruple regimen was $96 \%$, a very high rate. This result is largely due to the instructions and information concerning the drug and its use that were informed to all the patients.

While determining potential risk factors associated with failed eradication, we noted that the treatment efficacy decreased significantly with older group ranges. A similar finding was observed in a study from Southern Iran that showed a meaningful difference in eradication for elderly patients (and mainly men) [21].

This association might be due to several possible reasons that need to be elucidated: high gastric acidity, antibiotic resistance, high bacterial load, and the burden of polypharmacy.

However, in our study, none of the factors including patient's gender, treatment assignment, had a positive association with the eradication rate. This result was also seen in a study on a Chinese population where no factors such as gender, smoking were associated with failure of eradication [5].

\section{Limitations}

The limitations to our study would be the selection of a relatively small sample size and the choice of the population enrolled which was only from one hospital belonging to the same region. This could have resulted in a selective representation rather than a homogenous one. Consequently, our results cannot be reliably extrapolated to the entire Lebanese population.

In order to overcome this limitation, a study should be made on a broad area of our country, recruiting patients from different hospitals.

Other studies should be done to evaluate the antibiotics resistance especially to Clarithromycin, metronidazole and Amoxicillin in a large population in Lebanon in order to adjust the treatment of $H$ Pylori accordingly.

\section{Conclusion}

In summary, H. pylori is associated with several diseases whether digestive or extra digestive. Despite the several combinations used to treat this infection, the complete eradication is not possible yet.

However, the combination formulation of the non-bismuth quadruple therapy achieved a high eradication rate $94 \%$ with a $96 \%$ compliance. An association between age and failure of eradication has been noticed. Although it might be due to antibiotic resistance, high bacterial load or high acidity among others; this treatment failure should be studied further.

Finally, and based on the evidence presented in this prospective randomized study we concluded that the concomitant therapy given for 14 days is effective and safe, as one of the first-line treatment options for eradicating $H$. pylori infection.

\section{References}

1. Ruggiero P (2010) Helicobacter pylori and inflammation. Current Pharmaceut Design 16: 4225-4236. [Crossref]

2. Kusters JG, van Vliet AH, Kuipers EJ (2006) Pathogenesis of Helicobacter pylori Infection. Clin Microbiol Rev 19: 449-490. [Crossref]

3. Yi-Jian D, Tian-Lian Y, Xin-Lan H, Jian-Hua L, Chao-Hui Y, et al. (2015) Association of Salivary Helicobacter pylori Infection with Oral Diseases: a Cross-sectional Study in a Chinese Population. Int J Med Sci 12: 742-747. [Crossref]

4. Park SH (2015) Changes in upper gastrointestinal diseases according to improvement of Helicobacter Pylori prevalence rate in korea. Korean J Gastroenterol 65: 199-204. [Crossref]

5. Liu KS, Hung IF, Seto WK, Tong T, Hsu AS, et al. (2014) Ten day sequential versus 10 day modified bismuth quadruple therapy as empirical firstline and secondline treatment for Helicobacter pylori in Chinese patients: an open label, randomized crossover trial. Gut 63: 1410-1415. [Crossref]

6. Kobayashi I, Murakami K, Kato M, Kato S, Azuma T, et al. (2007) Changing Antimicrobial Susceptibility Epidemiology of Helicobacter pylori Strains in Japan between 2002 and 2005. J Clin Microbiol 45: 4006-4010. [Crossref]

7. Kim SY, Choi DJ, Chung JW (2015) Antibiotic treatment for Helicobacter pylori: Is the endcoming? World J Gastrointest Pharmacol Ther 6: 183-198. [Crossref]

8. Chey WD, Leontiadis GI, Howden CW, Moss SF (2017) Treatment of Helicobacter pylori infection. Am J gastroenterol 112: 212-239.

9. Jheng GH, Wu IC, Shih HY, Wu MC, Kuo FC, et al. (2015) Comparison of SecondLine Quadruple Therapies with or without Bismuth for Helicobacter pylori Infection. BioMed Res Int: 163960. [Crossref]

10. Essa AS, Kramer JR, Graham DY, Treiber G (2009) Meta-analysis: Four-Drug, ThreeAntibiotic, Non-bismuth- Containing "Concomitant Therapy" Versus Triple Therapy for Helicobacter pylori Eradication. Helicobacter 14: 109-118. [Crossref]

11. Saleem A, Qasim A, O'Connor HJ, O'Morain CA (2009) Pylera for the eradication of Helicobacter pylori infection. Expert Rev Anti Infect Ther 7: 793-799. [Crossref]

12. Laine L, Hunt R, El-Zimaity H, Nguyen B, Osato M, et al. (2003) Bismuth-based quadruple therapy using a single capsule of bismuth biskalcitrate, metronidazole, and tetracycline given with omeprazole versus omeprazole, amoxicillin, and clarithromycin for eradication of Helicobacter pylori in duodenal ulcer patients: a prospective, randomized, multicenter, North American trial. 2003. Am J Gastroenterol 98: 563-567. [Crossref] 
13. O'Morain C, Borody T, Farley A, De Boer WA, Dallaire C, et al. (2003) Efficacy and safety of single-triple capsules of bismuth biskalcitrate, metronidazole and tetracycline, given with omeprazole, for the eradication of Helicobacter pylori: an international multicentre study. Alimentary Pharmacol Ther 17: 415-420. [Crossref]

14. He L, Deng T, Luo H (2015) Meta-Analysis of Sequential, Concomitant and Hybrid Therapy for Helicobacter pylori Eradication. Intern Med 54: 703-710. [Crossref]

15. Saeed EA, Rosenthal KJ, David G, Gerhar T (2010) Meta-analysis: Four drug,three antibiotic, non- bismuth containing concomitant therapy vs. triple therapy for helicobacter Pylori eradication. Helicobacter 14: 109-118. [Crossref]

16. McColl KEL (2012) Clinical practice: Helicobacter pylori infection. Gastroenterol Hepatol 8: 621-623.
17. Jung SM, Cheung DY, Kim JI, Kim I, Seong H (2015) Comparing the Efficacy of Concomitant Therapy with Sequential Therapy as the First-Line Therapy of Helicobacter pylori. Gastroenterol Res Pract 2016:1-5.

18. Chang SS, Hu HY (2015) Helicobacter pylori: Effect of coexisting diseases and update on treatment regimens. World J Gastrointest Pharmacol Ther 6: 127-136.

19. Safavi M, Sabourian R, Foroumadi A (2016) Treatment of Helicobacter pylori infection Current and future insights. World J Clin Cases 4: 5-19.

20. Jimmy B, Jose J (2011) Patient medication adherence: measures in daily practice. Oman Med J 26: 155-159. [Crossref]

21. Masoodi M, Panahian M, Rezadoost A, Heidari A (2013) Eradication rate of Helicobacter pylori using a Two-week quadruple therapy: A report from Southern Iran. Middle East J Dig Dis 5: 81-85. [Crossref]

Copyright: (C2020 Ali K. This is an open-access article distributed under the terms of the Creative Commons Attribution License, which permits unrestricted use, distribution, and reproduction in any medium, provided the original author and source are credited. 